\title{
Speaker Abstracts from the ASENT 2005 Annual Meeting March 3-5, 2005
}

\section{The View from the Pharmaceutical Industry}

\author{
Roger J. Porter, M.D.
}

The era of the early 1990s was characterized by a burst of approvals of new antiseizure drugs. Although many of the older drugs such as phenytoin, carbamazepine and valproate have maintained very substantial market share, the contribution of many of the newer drugs has been, for many individual patients, a significant improvement in seizure control, adverse effects, and quality of life.

How can we sustain (or recapture) this successful era, and bring even better, more effective, less toxic antiseizure drugs to the patients who are in need? What are the variables in play that determine whether or not we can expect a continuing flow of new drugs, and what are the factors that work against us in this quest? This lecture will address these issues and will describe in some detail the pharmacoeconomic aspects of the pharmaceutical industry; it will expand on these issues within the industry that affect our specific area of interest. Where epilepsy stands in the hierarchy of drugs under study will be reviewed.

One of the specific variables that must be addressed is the use of new antiseizure drugs for indications other than epilepsy. Such usage should not come as a surprise to older neurologists. After all, phenobarbital was first brought to the marketplace for tranquilization and sedation; epilepsy was a serendipitous afterthought. Phenytoin, though first developed for epilepsy, had numerous other disorders attached to it such as pain and cardiac arrhythmias. Carbamazepine was first marketed in the U.S. for trigeminal neuralgia - I can remember, as a resident physician in the early 1970 s, being criticized for using it on my patients with epilepsy. In this new era, it is clear that epilepsy is not at the forefront of desirable markets for most of the largest pharmaceutical companies, and a "different hook" is needed. We may be well served by finding non-epilepsy indications for potential new antiseizure drugs; such will potentially broaden the market, increase the yield to the company and cause larger companies to take notice of the drug, even in early stages of development. We need to emphasize the opportunities in neuropathic pain, migraine, bipolar disorder and other common disorders. We can do this with screening programs for earlystage drugs and with proof-of-principle studies in later development stages. A broader indication list can sometimes take a marginal market performer and make it into a major sales success.

Another curious factor is the misperception that epilepsy alone is an inadequate market. Many of the newer antiseizure drugs are yielding several hundreds of millions of dollars of sales from epilepsy sources alone; some are true blockbusters.

Other areas of interest will be considered. What is the role of epilepsy as a niche disorder for a small company? In pediatrics, the 6-month exclusivity rule has been extraordinarily successful in the creation of studies in children. How can we best obtain adequate and timely pediatric studies in future drug development studies so that data for pediatricians is not indefinitely delayed?

The challenges to the pharmaceutical industry are inextricably interrelated to the questions of availability of new antiseizure drugs. This lecture will relate these challenges to the potential for improved health care for our patients with epilepsy.

\section{AEDs Outside of Epilepsy-Case Study: Divalproex for Mania}

Kenneth Sommerville, M.D.

The use of antiepilepsy drugs (AEDs) for conditions other than epilepsy represents a potentially large area to serve multiple unmet medical needs. AEDs have been approved for mania, depression, migraine, and certain categories of neuropathic pain. The approval of an AED for a new indication offers both challenges and opportunities. An understanding of these issues enables more effective planning for drug development and hopefully a better product for the Sponsor, medical community, and patients.

An early example of an AED approved in a completely new therapeutic area was the approval of divalproex (Depakote ${ }^{\circledR}$ ) for acute mania of bipolar disease. Filing for use of divalproex in this indication was in 1992. Approval was granted in 1995.

The approval of divalproex for mania was influenced by the:

- existing approval in epilepsy

- evidence of efficacy

- inherent risks of further development

- feasibility of obtaining approval

- existing label of epilepsy

Although specifically important to the divalproex program, each of these factors are important for approval of any marketed drug for a new indication.

The program of divalproex in mania was a success and the pattern of development is now a part of many new AED programs. The more aggressive approach of including multiple indications at the beginning of the program of a new chemical entity (NCE) is an example of the progression of this concept.

Lacosamide (LCM) is an NCE with an unknown mechanism of action now in phase 3 for both adjunctive treatment of partial seizures and diabetic neuropathic pain. Key issues for approval in these indications are similar to the experience with divalproex but there is no previously approved labeling or post-marketing experience to guide the development. Considerations for development of these two indications in parallel are:

- number of exposures needed for each possible indication for safety/efficacy

- effective doses for each indication

- which assessments for safety may support all indications

- similarities and differences between the population of each indication

- which interaction studies are needed and which pharmacology studies support all indications

- which formulations are appropriate for each indication (tablets, syrup, intravenous)

In summary, AEDs are useful in multiple areas outside of epilepsy so development for these indications makes sense both 
medically and as a business decision. Some efficiencies are possible in manufacturing, preclinical, and biopharmaceutics but specific and large programs are still needed for each indication. The development of AEDs outside of epilepsy is now a routine part of AED development.

Reference: SBA for Depakote ${ }^{\circledR}$ in acute mania of bipolar disease, 1995.

\section{Running a Patient-Oriented Research Program for Residents}

Jacqueline A. French, M.D.

There are many reasons why patient-oriented research training should begin during residency.

Firstly, introducing residents to patient-oriented research methodology and evidence based medicine is a crucial step in their ability to critically review the medical literature in order to provide better patient care. This would be important for all residents, whether their future plans include research, or clinical neurology. Clinical trials form the basis of the evidence by which we select therapies, determine prognosis, and make diagnoses. Residents that do not understand research methodology may make inappropriate conclusions from the literature.

A second reason is to provide information so that motivated residents can plan for their careers post residency. There is a great need for well-trained researchers in this area. In the current academic environment, appropriate training is an absolute requirement before launching on a career in patient-oriented research. An introduction to patient-oriented research during residency will allow residents to understand the rigors of a patient-oriented research career, and hopefully will attract a proportion of "the best and the brightest" into this career path.

At the University of Pennsylvania, we have created a formal patient-oriented research program for all residents receiving training at our institution. This program has a number of components. There is a semiweekly patient-oriented research conference. For each session, a resident is paired with a faculty member who is knowledgeable in patient-oriented research. Together, they select an article from the current literature. Using guides from the McMasters Evidence Based Medicine Series, they critically review the article, and the resident then leads a discussion at the conference. An attempt is made to sequentially address major areas of patient-oriented research. Each resident is also required, in partnership with a faculty mentor, to undertake a limited research project, which can be either clinical or basic in nature. They are required to prepare a research proposal. This proposal must then be presented to their peers, who critique it. In this way, the residents are exposed to peer review. Once the research is undertaken, it is ultimately presented to the assembled faculty.

Our patient-oriented research program has been very successful. Many of the residents have submitted their research for publication, or have continued their research project into fellowship. Most tell us that this program was a very rewarding aspect of their training. We urge other neurology residency programs to consider incorporating a patient-oriented research program into their training experience.

\section{Clinical Disorders of GABA Metabolism}

$$
\text { Phillip Pearl, M.D. }
$$

Amino acid neurotransmitters carry the major "yes/no" commands in the CNS, as opposed to the modulatory roles of other transmitters. Up to $1 / 3$ of brain synapses employ GABA, the major inhibitory neurotransmitter. Its major precursor is L- glutamate, which is converted to GABA via glutamate decarboxylase (GAD). The GABA shunt is a closed loop that involves transamination of $\alpha$-ketoglutarate to glutamate, which is then converted via GAD to GABA. GABA is transaminated to succinic semialdehyde via GABA-transaminase (GABA-T), which requires the presence of $\alpha$-ketoglutarate to accept the amine group. Thus, this restores glutamate, and a molecule of the GABA precursor is formed as a molecule of GABA is catabolized. The glutamine-glutamate or glial-neuronal shuttle is an ancillary loop. Released GABA is taken up by glial cells, where glutamate can be formed but not converted to GABA due to absence of GAD. Instead, GABA is converted via glutamine synthetase to glutamine, which is returned to the neuron and via glutaminase is converted to glutamate. Thus, the loop is completed and the supply of GABA precursor is conserved. The synthesis of GABA via GAD requires the coenzyme pyridoxal-5-phosphate (PLP). Pyridoxine-dependent epilepsy is classically a neonatal syndrome but potentially more common presentations, with later and atypical features, widen the phenotype. While traditionally considered a disorder of GABA synthesis, no consistent abnormality of GABA metabolism or GAD has been identified and other PLP-dependent pathways are implicated. Succinic semialdehyde dehydrogenase (SSADH) is required for the conversion of succinic semialdehyde to succinic acid, and SSADH deficiency leads to accumulation of g-OH-butyric acid (GHB) in physiological fluids. GHB is a $\mathrm{GABA}_{\mathrm{B}}$ receptor agonist, inhibits presynaptic dopamine release, and may function independently as a neurotransmitter. SSADH deficiency is characterized by severe language deficits, ataxia, and hyporeflexia. Increased detection is reported when specific ion monitoring is used for GHB on urine organic acids. The index case was described by Jakobs in 1981, and since then over 350 patients have been identified. We have a database of 91 patients, including 44 from detailed published reports and 47 from systematic questionnaire data. The clinical features are developmental delay (100\%), mental retardation $(81 \%)$, hypotonia $(64 \%)$, ataxia $(53 \%)$, and seizures $(47 \%)$. The disorder has the course of a nonprogressive encephalopathy, rendering the index of clinical suspicion for a metabolic disorder low. Prominent behavior problems include sleep disturbances, aggression, inattention, hyperactivity, and hallucinations. Among the patients with seizures, the most common seizure types are generalized tonic-clonic (59\%), absence (45\%), and myoclonic (23\%). EEG features include background abnormalities in $20 \%$ and epileptiform discharges (usually generalized) in $32 \%$. MRI abnormalities include cerebral atrophy $(11 \%)$, cerebellar atrophy $(7 \%)$, and increased $\mathrm{T} 2$ weighted signal involving globus pallidus (43\%), dentate nucleus (18\%), subcortical white matter $(7 \%)$, and brainstem (7\%). MR spectroscopy has demonstrated the first example of increased endogenous GABA in human brain parenchyma in this disorder. GABA-transaminase deficiency and homocarnosinosis are very rare but require CSF for detection, thus allowing for the possibility that these entities, as in the other pediatric neurotransmitter disorders, are underrecognized.

\section{Pediatric Epilepsy Clinical Trials}

Blaise F.D. Bourgeois, M.D.

I. Pediatric Differences with Potential Impact on Drug Trials In order to address the issue of pediatric epilepsy clinical trials as a separate entity from trials in adults, the first step is to delineate how epilepsy in children differs from epilepsy in adults. Children have different epilepsies and seizure types; the differential diagnosis of their seizures is broader; the etiologies 
of their epilepsies are different; the decision to treat after first seizure may differ; children may receive treatments not used in adults; several antiepileptic drugs (AEDs) are used off-label in children; children have different pharmacokinetics; children may have different adverse effects to AEDs; epilepsy in children is more likely to remit; seizure relapse is less likely after drug discontinuation.

Certain differences in children that may have a potential impact on AED drug trials have been well documented. These include different pharmacokinetics in children, their different seizures and epilepsy syndromes, different age-related side effects, different co-morbidities, and a greater risk of seizure exacerbation. All of these factors can potentially add a level of complexity to AED trials in the pediatric age range. There is also the general perception that children are a more vulnerable population that needs to be protected. However, the lack of controlled trials in children is more likely to have an opposite effect, which is to expose them to the risk of off-label use of medications for which no carefully collected data are available.

II. Available Evidence from Pediatric Trials

Several evidence-based guidelines on various aspects of the treatment of epilepsy have been developed in the recent past. These publications provide an extensive review of pediatric epilepsy clinical trials. These reviews highlight the overall lack of class I evidence in the drug treatment of seizures and syndromes that are more specific to the pediatric age range. For instance, there is no class I study for any AED as a first drug in untreated juvenile myoclonic epilepsy. For infantile spasms, one treatment has been rated as "probably effective" (ACTH) and another as "possibly effective" (vigabatrin). There was not sufficient evidence to recommend any other treatment. Also, the paradigm for treatment of neonatal seizures has remained the same for several decades.

III. Future Directions

There is a substantial need for more evidence-based data in pediatric epilepsies and epilepsy syndromes. The pharmaceutical industry should be encouraged to support pediatric AED trials early in the development. RCTs should remain the norm in pediatric AED trials, but they may have to be tailored to the particular patient population under study. Recruitment into RCTs is more difficult once a drug is on the market.

\section{Maintaining the Best Interest of the Patient: Careers in the Research Oriented Pharmaceutical Industry}

Mark Corrigan, M.D.

The development of improved therapeutic agents is critical to the advancement of medicine and welfare of patients. The Research based pharmaceutical industry has been responsible for the vast majority of new medicines currently available. The development of those medicines requires the collaboration of academic centers, the NIH, investigators and patients. Physicians wishing to make the broadest possible impact on the improvement on public health through the development of novel medicines have a variety of career choices within the pharmaceutical industry. These range from bench activities elucidating potential mechanisms (including genomics), development of biomarkers through experimental medicine divisions, clinical pharmacology, design and implementation of drug development plans through clinical research, project management, regulatory interfaces with the FDA and finally commercial roles. The public view of the pharmaceutical industry has changed over the last 5 years from one of admiration for a profitable business with lofty aspirations, to one that is now viewed as profiteering at the expense of the elderly and placing patients at undue risk with their products. This shift in view with subsequent financial consequences for companies, coupled with spiraling research costs bodes poorly for the next generation of therapeutics and for physicians concerned for the improvement in pharmaceutical treatments. Repairing the covenant with patient, while maintaining the balance between profit driven enterprise and commitment to research is the challenge for the pharmaceutical industry and physicians within it for the next decade.

\section{Neuroimaging and Therapeutics in Movement Disorders}

David Eidelberg, M.D.

Movement disorders represent a group of syndromes characterized by an impairment of the regulation of voluntary motor activity without deficits of force, cerebellar function, or sensation. This class of neurological diseases includes hypokinetic disorders associated with a slowing of movements such as Parkinson's disease (PD), as well as hyperkinetic disorders characterized by involuntary abnormal movements such as Huntington's disease (HD), torsion Dystonia, and tic disorders. Generally, the clinical manifestations of movement disorders result from dysfunction of the basal ganglia. Although histopathological studies reveal specific neurodegenerative changes in some of these diseases (e.g., PD and HD) the pathological basis for many movement disorders remains unknown. Various imaging techniques have been used to visualize pathological changes in these disorders. Radiotracer imaging techniques using PET and SPECT imaging can be used to evaluate and quantify changes in specific neurochemical systems. Alternatively, disease-related changes in local brain function can be assessed with generalized traces for regional cerebral metabolism and blood flow.

Brain imaging in movement disorders was originally introduced to visualize the pathological changes associated with different clinical syndromes. Subsequently, these techniques have been utilized in longitudinal studies designed to assess disease progression and the effects of potential neuroprotective strategies. Lately, functional imaging has also been applied in the objective assessment of symptomatic treatment responses. In addition to providing an objective descriptor of the treatment response, brain imaging can also link clinical outcome to alterations in regional brain function.

We will describe the functional neuroimaging strategies to evaluate and monitor therapeutic interventions for movement disorders. Because PD is the most common and broadly studied of these disorders, our discussion will focus on the use of imaging to assess treatment options for this condition.

\section{Novel Approaches to Imaging of Brain Tumors: Lessons for Clinical Research Initiatives}

\author{
Fred Hochberg, M.D.
}

For Neuro-Oncologists, MRI offers phase I endpoints of neurologic toxicity. The assessment of responders in Phase II trials utilizes RECIST measures of one-dimension tumor size. These measures are plagued by shortcomings: They take months to utilize and offer little insight into changes in the biology of primary brain malignancy. They supply little information as to changes in "neovascularity" nor reduction of vascular permeability nor improvements in cerebral edema.

Novel therapies mandate novel imaging approaches. The specific examples of five such techniques will be provided: 1) 
Modulators of cell-cycle specific determinants of angiogenesis (such as EMD or Iressa) require rapid sequence MRI studies to provide measures of changing blood supply to tumors (Cerebral blood volume or Cerebral blood flow). 2) Drugs (glucocorticoids) which change the nature of endothelial tight junctions likely stabilize the "permeability" of tumor associated vessels. 3) The effectiveness of intra-tumoral novel gene therapies (retroviral, adenoviral and oncolytic herpes) can be measured by analysis of changes within the injection sites as distinct from the main tumor mass. 4) Tumor associated alterations in white matter can be assessed by white-matter sensitive MR sequences. 5) Nomograms of MR "response" of tumor volume, built for cohorts of patients with brain lymphoma who are cured by Methotrexate administration, can be utilized to separate early responders from early failures.

These novel approaches, often with in situ measures of drug concentrations, can easily be applied across a broad band of therapies. MR-based initiatives can be applied to studies of parkinsonism, leukoencephalopathy and dementia.

\section{Measurement of CSF Neurotransmitter Metabolites for Diagnosis of PNDs}

Keith Hyland, Ph.D.

The investigation of infants and children with suspected pediatric neurotransmitter diseases (PNDs) affecting serotonin and catecholamine (dopamine and norepinephrine) metabolism is complicated by the fact that measurement of metabolites in peripheral fluids is generally uninformative. Disorders affecting catecholamine and serotonin neurotransmission, that do not present with hyperphenylalaninemia, require that a lumbar puncture be performed and that specific metabolites be assessed in the collected cerebrospinal fluid (CSF). Monoamine neurotransmitter metabolites (homovanillic acid and 5-hydroxyindoleacetic acid), tetrahydrobiopterin and neopterin measurement is necessary to identify several disorders including dominantly inherited GTP cyclohydrolase deficiency, and the deficiencies of sepiapterin reductase, tyrosine hydroxylase and aromatic L-amino acid decarboxylase. Characteristic changes in neurotransmitter metabolites can also be seen in inherited disorders affecting the metabolism of pyridoxal 5'-phosphate. In addition, a marker for a folinic acid responsive, intractable neonatal seizure disorder can be detected on the HPLC system used to measure CSF neurotransmitter metabolites. Many of these marker compounds are labile, and this together with the presence of rostro-caudal gradients and age related changes in metabolite concentrations requires that strict sample collection and handling procedures be adhered to if meaningful results are to be obtained. The symptoms in the GTPCH deficiencies are often completely treatable and in the other conditions therapy can certainly improve quality of life both for the affected patients and their families. It is therefore important that awareness of this group of disorders be expanded and that CSF testing become routine in patients with appropriate symptoms and signs.

\section{Research and the Neuro-Cognitively Impaired Child}

Robert M. Nelson, M.D., Ph.D.

The inclusion of individuals in research who have neurocognitive impairments that may affect decision-making capacity has been addressed by The National Commission (1978) and the National Bioethics Advisory Commission (NBAC, 1998). The National Commission recommended that such research could be performed ethically, provided that additional protections were in place to limit participant exposure to the burdens and risks of research. However, the proposed regulations on research involving the "mentally disabled" were never adopted. As a result, the "Common Rule" (Subpart A) governing the participation of human subjects in research does not address the inclusion of individuals with neuro-cognitive impairments. The National Commission's recommendations concerning research involving children were adopted, affording children the additional protections now found in Subpart D of the federal regulations. However, the documented wide variability in the interpretation of "minimal risk" and the lack of concrete guidelines on child assent undermine the effectiveness of these additional protections. In addition, children with neuro-cognitive impairment may be more vulnerable to exposure to inappropriate research risk based on such factors as their residential setting or guardianship status.

This presentation will review the current regulations governing research involving children (i.e., Subpart D), and the issues raised when children with neuro-cognitive impairments are included in research. Along with the work of The National Commission and NBAC, the recent (March 2004) report of the Institute of Medicine on the Ethical Conduct of Research Involving Children will be reviewed, focusing on the interpretation of minimal risk, the inclusion of children with a condition or disorder, and child assent. Finally, the perceived vulnerability of children with neuro-cognitive impairments will be addressed in the context of selected state regulations and court cases, such as the 2001 decision by the Maryland Court of Appeals. 